\section{Influência regional no consumo precoce de alimentos diferentes do leite materno em menores de seis meses residentes nas capitais brasileiras e Distrito Federal}

\author{
Regional influence on early consumption of foods \\ other than breast milk in infants less than 6 months \\ of age in Brazilian State capitals and the Federal \\ District
}

\author{
Silvia Regina Dias Medici Saldiva 1 \\ Sonia Isoyama Venancio 1 \\ Ana Gabriela Cepeda Gouveia 1 \\ Ana Lucia da Silva Castro 1 \\ Maria Mercedes Loureiro Escuder 1 \\ Elsa Regina Justo Giugliani 2
}

\footnotetext{
1 Instituto de Saúde, Secretaria de Estado da Saúde de São Paulo, São Paulo, Brasil. 2 Universidade Federal do Rio Grande do Sul, Porto Alegre, Brasil.

Correspondência S. R. D. M. Saldiva Núcleo de Nutrição, Instituto de Saúde, Secretaria de Estado da Saúde de São Paulo. Rua Santo Antonio 590 3o andar, São Paulo, SP 01314-000, Brasil. smsaldiva@isaude.sp.gov.br
}

\begin{abstract}
The aim was to assess regional influences on food consumption in infants less than six months of age. A cross-sectional study was conducted in a sample of 18,929 infants participating in the Second Survey on Breastfeeding Prevalence in Brazilian State Capitals and the Federal District in 2008. Consumption rates for tea, fruit juices, formula milk, and porridge were calculated for the State capitals from the five geographic regions of the country. Food consumption was estimated by logit analyses and Poisson models. Differences in food consumption profile were observed between the different regions: tea was more common in State capitals in the South $(R P=2.37)$, while nonmaternal milk $(R P=1.50$ and 1.47$)$ and juices $(R P=1.57$ and 1.55$)$ were more frequent in the Northeast and Southeast, respectively. Porridge was more common in the Northeast $(R P=3.0)$. Brazil's geographic regions thus display different infant feeding patterns. Public policy should take cultural diversity into account when planning strategies to improve infant nutrition and health.
\end{abstract}

Supplementary Feeding; Food Consumption; Infant

\section{Introdução}

A Organização Mundial da Saúde (OMS) recomenda, desde 2001, que os alimentos complementares sejam introduzidos na dieta da criança em torno dos seis meses de idade 1 . Kramer \& Kakuma ${ }^{2}$, em revisão sistemática quanto à época ideal para a introdução desses alimentos, concluíram que esta, antes dos seis meses, não só é desnecessária, como pode ser prejudicial, por aumentar o risco de doenças infecciosas e gastrointestinais. Além disso, a introdução precoce de alimentos pode levar à desnutrição, quando a oferta de alimentos não supre os requerimentos nutricionais 3 ; interferir negativamente no estabelecimento de hábitos alimentares saudáveis e propiciar riscos para o trato digestivo, vias respiratórias e função renal ${ }^{4}$. Pode, ainda, contribuir para a superalimentação e a obesidade infantil 5 .

Apesar das evidências favoráveis ao aleitamento materno exclusivo (AME) nos primeiros seis meses de vida, a prevalência dessa prática no Brasil ainda é baixa. Os resultados da II Pesquisa Nacional de Aleitamento Materno 6 mostraram que apenas $41 \%$ das crianças menores de seis meses de idade são amamentadas exclusivamente, corroborando outros estudos nacionais, que apontam frequência elevada de consumo precoce de chás, água, suco, outros leites e alimentos complementares 7,8,9,10,11.

Alguns fatores têm sido associados com a introdução precoce de alimentos exceto leite 
materno na dieta das crianças, tais como baixos níveis socioeconômico das famílias e educacional das mães, mães jovens, fumantes e parto cesáreo 8,10,12,13,14.

Tendo em vista a variação encontrada nas prevalências do AME em crianças menores de seis meses entre as regiões do país (de $37 \%$ no Nordeste a $45,9 \%$ no Norte), o presente estudo tem o objetivo de explorar as influências regionais sobre o consumo precoce de diversos alimentos em crianças de 0-6 meses de idade residentes nas capitais brasileiras e no Distrito Federal.

\section{Métodos}

Analisaram-se os dados de 18.929 crianças menores de seis meses participantes da II Pesquisa Nacional de Prevalência de Aleitamento Materno. Trata-se de uma pesquisa de corte transversal, realizada na segunda etapa da campanha de vacinação de 2008 em todas as capitais brasileiras e Distrito Federal 6.

Foram utilizadas amostras por conglomerados em dois estágios, sendo os planos amostrais elaborados com base nas informações da campanha de vacinação de 2007, fornecidas pelas Secretarias Municipais de Saúde de todas as capitais brasileiras. O tamanho amostral levou em consideração a prevalência de AME nas capitais e Distrito Federal em 1999, com acréscimo de 2\%$10 \%$, prevendo-se um aumento da prevalência. Para compensar as perdas de precisão inerentes à amostra por conglomerados, acrescentou-se o efeito do desenho, multiplicando-se por 1,5 a estimativa inicial e, ainda, uma taxa de não resposta de 5\%-10\%. O tamanho da amostra desejado para estimar o AME em menores de seis meses foi multiplicado por 26,15 .

As informações sobre as práticas alimentares foram coletadas por meio de recordatório de 24 horas, refletindo as práticas atuais de alimentação (current status). Em adição, foram colhidas informações sobre as crianças e suas mães, com a finalidade de analisar a alimentação segundo características da população. Todos os entrevistadores receberam treinamento para a aplicação do questionário e para a realização do sorteio sistemático das crianças nos postos de vacinação.

Cada município transferiu os dados obtidos para um aplicativo on-line, desenvolvido especificamente para a pesquisa. Posteriormente, o banco de dados de todas as capitais e Distrito Federal foi exportado para os programas SPSS 16.0 (SPSS Inc., Chicago, Estados Unidos) e Stata 10.1 (Stata Corp., College Station, Estados Unidos), a fim de se realizarem as análises. Para a confecção de tabelas e gráficos, foi utilizado o programa Excel 2003 (Microsoft Corp., Estados Unidos).

Neste estudo, as capitais brasileiras e o Distrito Federal foram agrupados de acordo com as cinco regiões geográficas do Brasil. Foram utilizados procedimentos específicos para análise de inquéritos com amostragem probabilística complexa, nos quais as estimativas de prevalência consideram o erro padrão, determinado pelo delineamento amostral, e seus respectivos intervalos de 95\% de confiança (IC95\%). Por causa das diferenças populacionais entre as capitais envolvidas, cada plano representou uma fração amostral diferente, representada pelo tamanho estimado da amostra sobre o número de crianças a serem vacinadas. O inverso dessa fração foi aplicado para o cálculo das prevalências regionais. Tendo em vista a opção de trabalhar com dados atuais sobre a alimentação infantil (current status), de acordo com recomendação da OMS 16, optou-se pela análise de logitos, realizada no programa SPSS, a qual permite, por modelagem estatística, a obtenção de curvas de probabilidades de consumo dos alimentos estudados conforme a idade em dias do conjunto de crianças da amostra.

As análises bivariadas e multivariadas foram realizadas no programa Stata 10.1 em virtude da opção por se utilizar modelos de Poisson com variância robusta, levando-se em conta os pesos amostrais. Considerou-se desfecho o consumo dos seguintes alimentos: chá, leite não materno (fórmula infantil, leite integral em pó ou fluido), sucos (natural e/ou artificial), mingau/papa (doce e/ou salgado). A região geográfica de residência da criança (Norte, Nordeste, Sudeste, Sul e Centro-oeste) foi considerada a principal variável independente ou de exposição, tendo como covariáveis, analisadas de forma dicotômica: sexo da criança (masculino; feminino), peso ao nascer $(<2.500 \mathrm{~g} ; \geq 2.500 \mathrm{~g})$, ordem de nascimento (primeiro filho; demais), escolaridade materna (< 8 anos de estudo; $\geq 8$ anos de estudo), condição de trabalho materno no momento da entrevista (trabalha fora; não trabalha fora/licença maternidade), tipo de parto (cesárea; normal/fórceps) e idade materna ( $\leq 20$ anos; 20 -35 anos; $\geq 35$ anos). $\mathrm{Na}$ análise multivariada, permaneceram no modelo final as variáveis que apresentaram $\mathrm{p}<$ 0,20 . Todas as análises foram controladas pela idade da criança em dias.

A pesquisa teve seu protocolo aprovado pelo Comitê de Ética do Instituto de Saúde da Secretaria de Estado da Saúde de São Paulo (protocolo $\mathrm{n}^{\circ}$. 001/2008 de 06/Mai/2008). 


\section{Resultados}

Do total de crianças estudadas, 9.779 tinham menos de três meses e 9.150 , de três a seis meses de idade. A Tabela 1 apresenta as principais características das mães e crianças.

Embora 94\% das crianças menores de três meses e $83 \%$ entre três e seis meses tenham consumido leite materno, a prevalência de AME em menores de três meses e entre três e seis meses foi de $52,2 \%$ e $25,4 \%$, respectivamente.

A Figura 1 mostra as frequências do consumo precoce de diferentes alimentos segundo faixa etária e região da capital de residência. Verifica-se que os outros tipos de leite aparecem com maior frequência, quando comparados aos demais alimentos, nas duas faixas etárias estudadas. Há um aumento expressivo na frequência de todos os alimentos quando comparadas as duas faixas etárias, exceto em relação ao consumo de chá. Chama atenção a maior frequência do uso de chá nas capitais da Região Sul, de suco e outros leites

Tabela 1

Características das mães e das crianças menores de seis meses de idade residentes nas capitais brasileiras e Distrito Federal, 2008

\begin{tabular}{|c|c|c|}
\hline \multirow[t]{2}{*}{ Características da população } & \multicolumn{2}{|c|}{$0-6$ meses } \\
\hline & $\mathbf{n}$ & $\%$ \\
\hline \multicolumn{3}{|l|}{ Sexo da criança } \\
\hline Masculino & 9.530 & 50,3 \\
\hline Feminino & 9.399 & 49,6 \\
\hline \multicolumn{3}{|l|}{ Peso ao nascer } \\
\hline$\leq 2.500 \mathrm{~g}$ & 1.477 & 8,2 \\
\hline$>2.500 \mathrm{~g}$ & 16.584 & 91,8 \\
\hline \multicolumn{3}{|l|}{ Primeiro filho } \\
\hline Sim & 8.309 & 49,6 \\
\hline Não & 8.448 & 50,4 \\
\hline \multicolumn{3}{|l|}{ Escolaridade da mãe } \\
\hline \multicolumn{3}{|l|}{ (anos de estudo) } \\
\hline$<8$ & 6.528 & 38,5 \\
\hline$\geq 8$ & 10.428 & 61,5 \\
\hline \multicolumn{3}{|l|}{ Trabalho da mãe } \\
\hline Trabalha fora & 5.379 & 33,4 \\
\hline Não trabalha fora & 10.726 & 66,6 \\
\hline \multicolumn{3}{|l|}{ Tipo de parto } \\
\hline Normal/Fórceps & 9.481 & 50,7 \\
\hline Cesárea & 9.223 & 49,3 \\
\hline \multicolumn{3}{|l|}{ Faixa etária da mãe (anos) } \\
\hline$<20$ & 3.089 & 18,3 \\
\hline $20-35$ & 12.071 & 71,4 \\
\hline$>35$ & 1.746 & 10,3 \\
\hline
\end{tabular}

nas da Região Sudeste e de mingau/papa nas da Região Nordeste.

Análise mais detalhada do consumo desses alimentos conforme idade pode ser visualizada na Figura 2. As curvas de probabilidade do consumo de chá, outros leites, sucos e mingau, segundo idade e capitais das diferentes regiões geográficas, mostram comportamentos distintos no consumo dos alimentos estudados. O consumo de chá nas capitais da Região Sul é maior que nas capitais de outras regiões em todas as idades; nas capitais do Nordeste, há maior consumo de mingau e, nas do Sudeste, verifica-se maior consumo de outros tipos de leite. Já em relação ao suco, as probabilidades de consumo até cerca de 40 dias de vida são muito semelhantes entre as regiões, e as diferenças só se distinguem a partir do segundo mês, quando as capitais das regiões Sudeste e Nordeste passam a apresentar consumo superior às demais. Por outro lado, as capitais da Região Norte apresentam o menor consumo de chá e outros leites, enquanto as do Centrooeste e Sul, o menor consumo de mingau, em todas as idades analisadas.

A Tabela 2 apresenta as estimativas das razões de prevalência bruta e ajustada para a associação entre o consumo dos diversos alimentos e as variáveis independentes. Observa-se que, utilizando o conjunto das capitais de uma determinada região que apresentou menor consumo de um alimento específico como linha de base, as capitais da Região Sul apresentaram maior razão de prevalência para o consumo de chá, ao passo que as capitais do Nordeste e do Sudeste, para o consumo de outros tipos de leite e de suco. As da Região Nordeste apresentaram razão de prevalência superior às das demais regiões no consumo de mingau/papa.

Além da influência regional, algumas das covariáveis estudadas mostraram-se associadas aos desfechos analisados. Introdução precoce de chá foi mais frequente em crianças do sexo masculino, primeiro filho e mães com pouca escolaridade; consumo de suco foi mais frequente em primeiro filho, cujas mães trabalhavam fora. Trabalho materno também se mostrou associado ao uso de outros leites, juntamente com baixo peso ao nascer. Já o consumo de mingau/papa foi mais comum entre as mães adolescentes e com baixa escolaridade.

\section{Discussão}

Embora os inquéritos populacionais realizados nas capitais brasileiras e Distrito Federal em 1999 e 2008 mostrem que a prática do AME vem aumentando 7, com acréscimo de 30,7 dias 
Frequência do consumo dos principais alimentos segundo faixa etária, em crianças menores de seis meses de idade das capitais brasileiras e do Distrito Federal, 2008.

1a) 0-3 meses

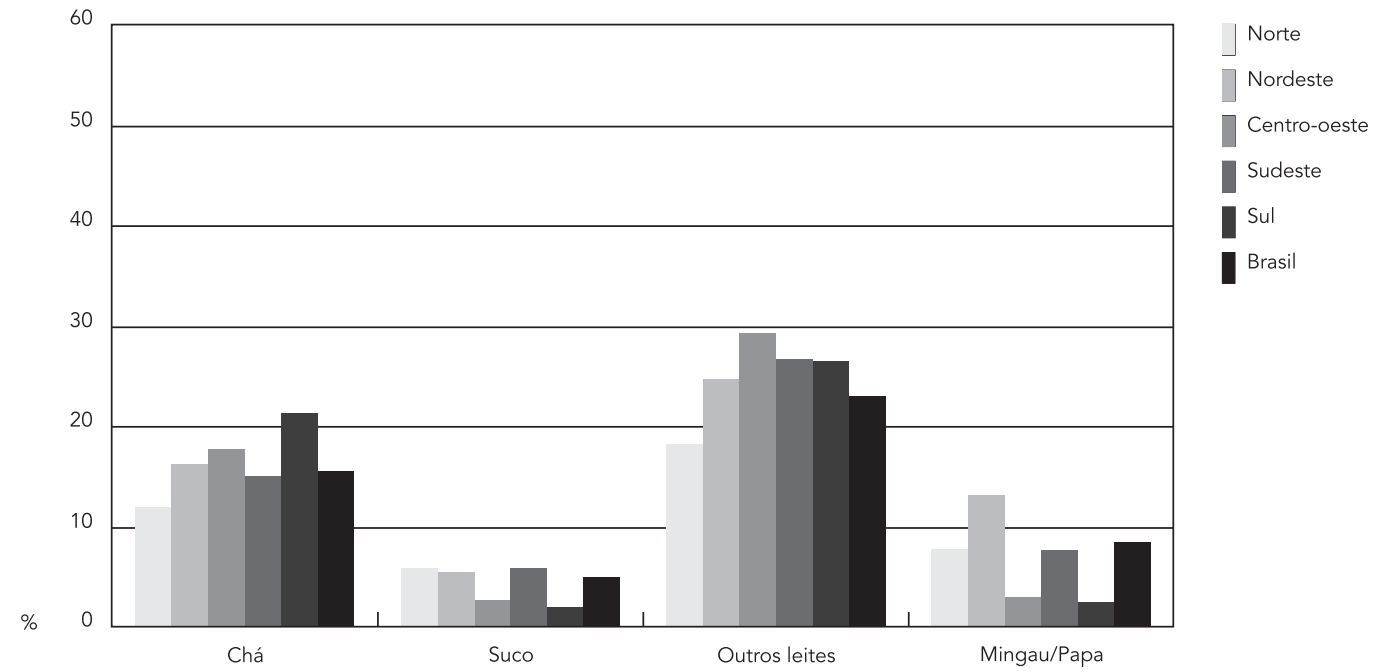

1b) 3-6 meses

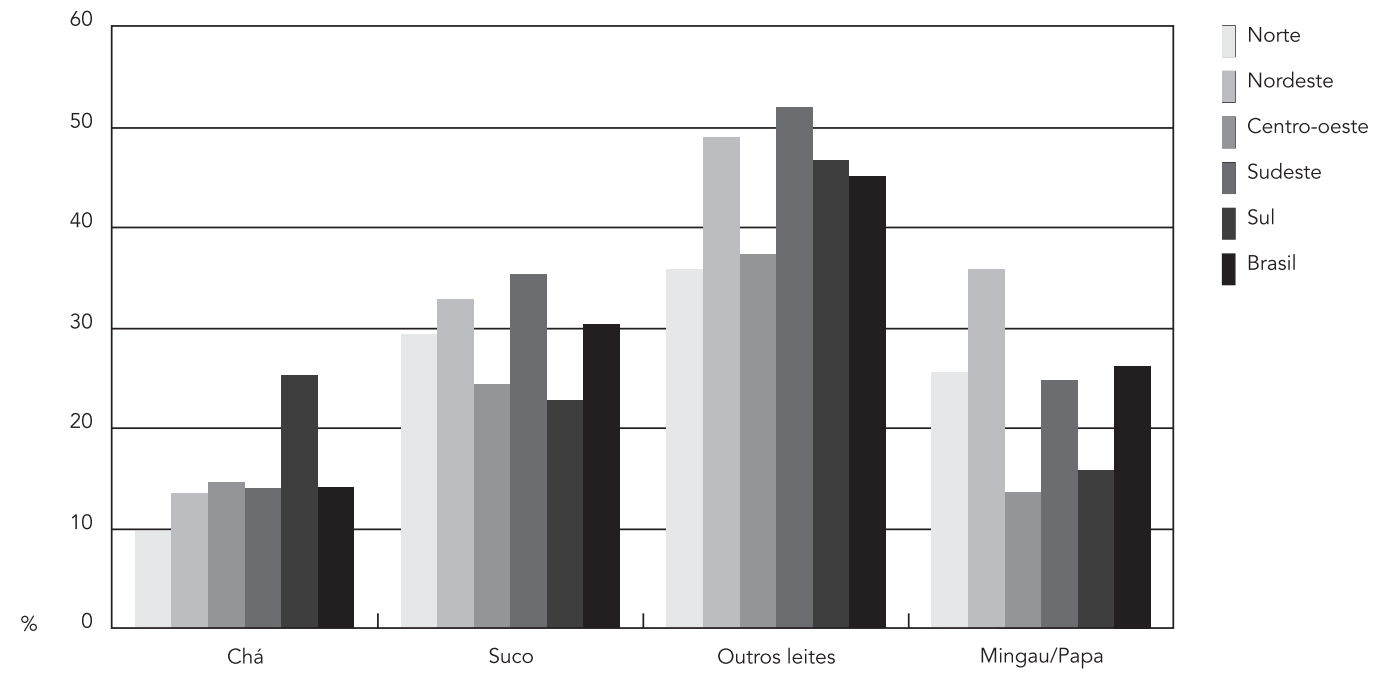

na sua duração mediana, o consumo precoce e desnecessário de alimentos diferentes do leite materno por crianças nos primeiros seis meses de vida continua sendo bastante frequente, corroborando os resultados de estudos nacionais e internacionais 6,10,11,13,17,18,19.

O presente estudo avaliou o consumo alimentar restrito às últimas 24 horas que antece- deram a pesquisa, sendo possível traçar, evitando possíveis vieses de memória, um perfil sobre o consumo de alimentos em crianças menores de seis meses. Porém, por se tratar de um inquérito realizado nas capitais brasileiras e Distrito Federal, não é possível generalizar os resultados aqui apresentados para as regiões como um todo, especialmente para as áreas rurais. Pode-se afir- 
Figura 2

Probabilidade do consumo de chá, outros leites, sucos e mingau/papa entre crianças menores de seis meses de idade residentes nas capitais brasileiras e Distrito Federal, 2008.

2a) Outros leites

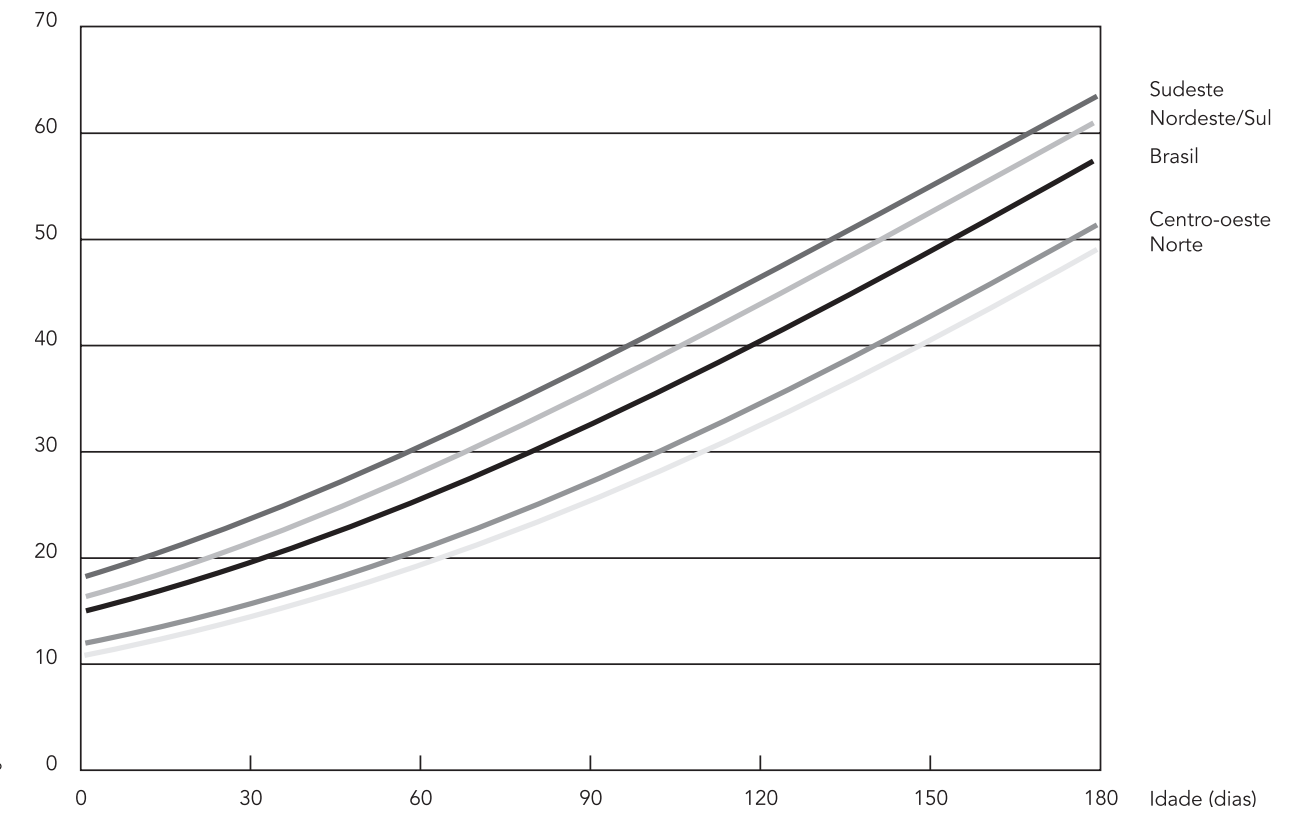

2b) Mingau

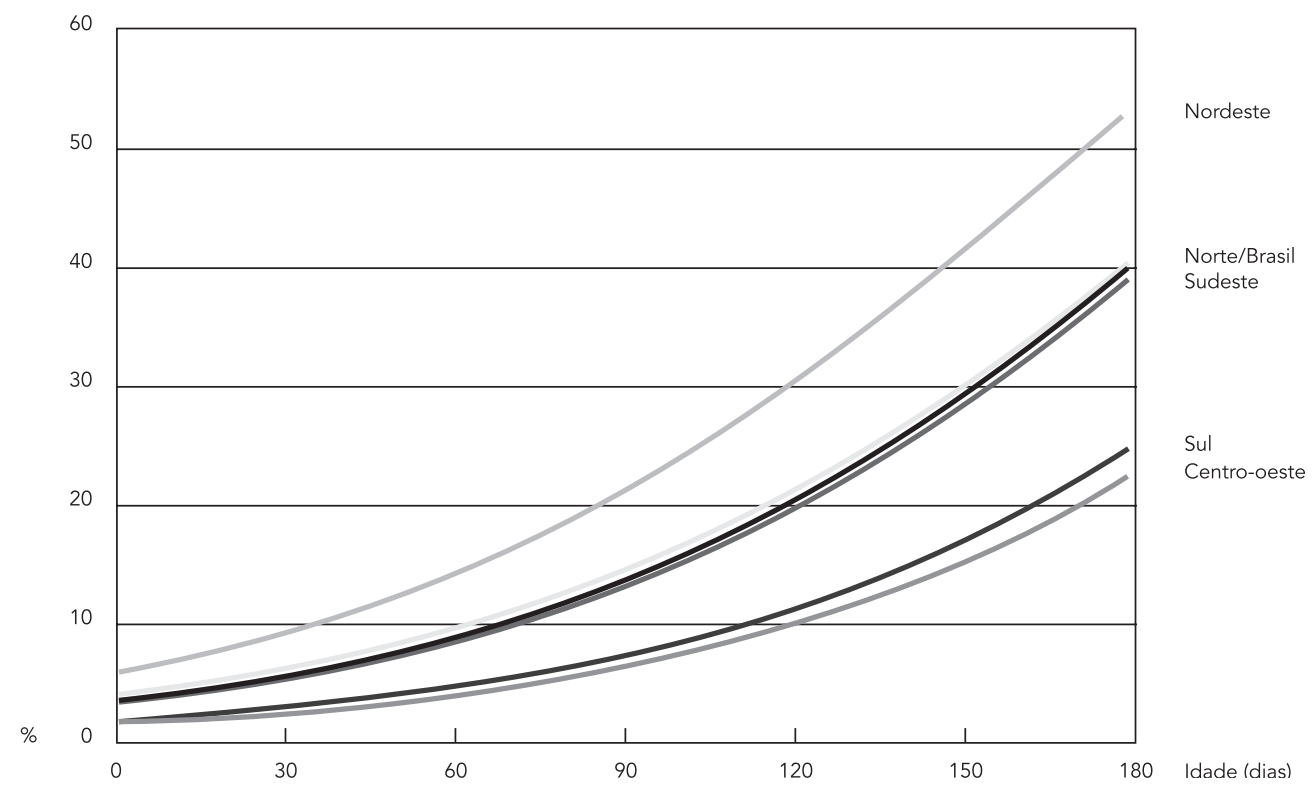


Figura 2 (continuação)

2c) Suco

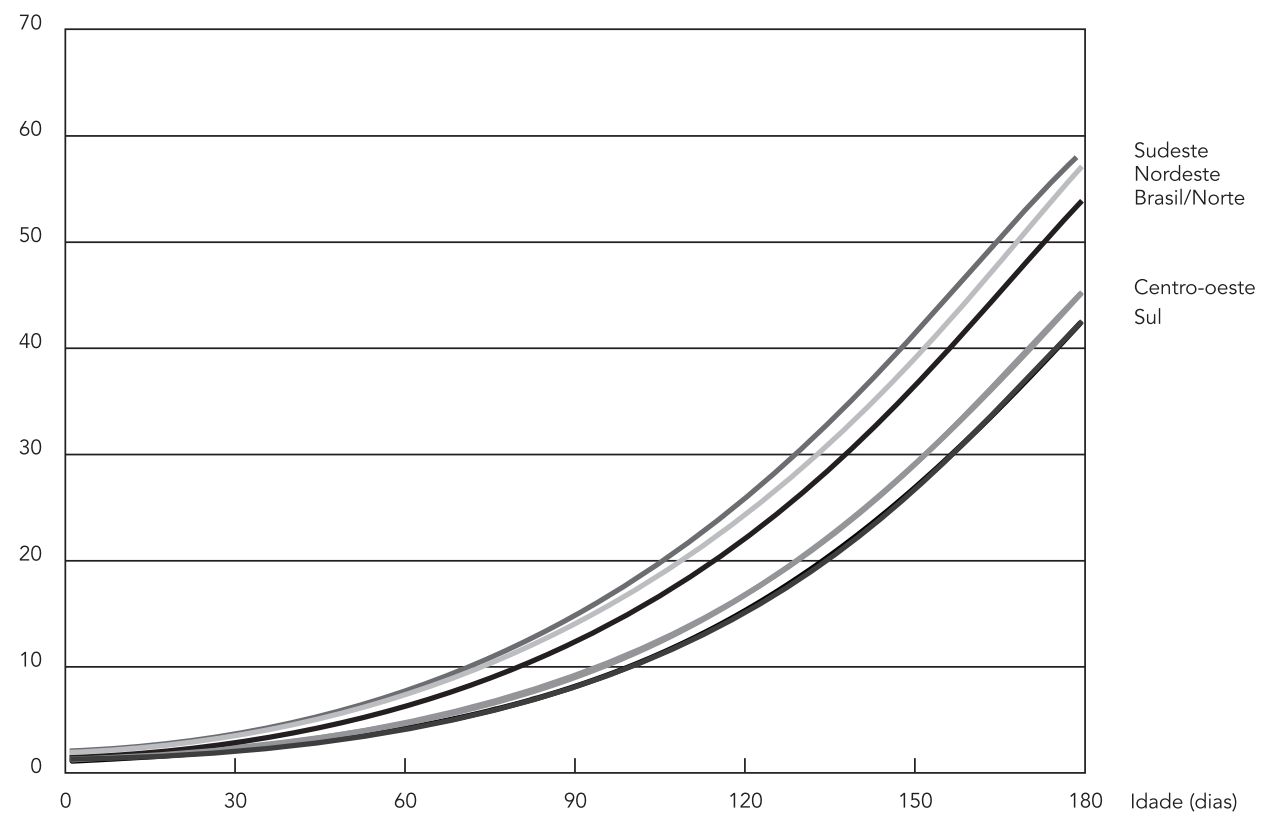

2d) Chá

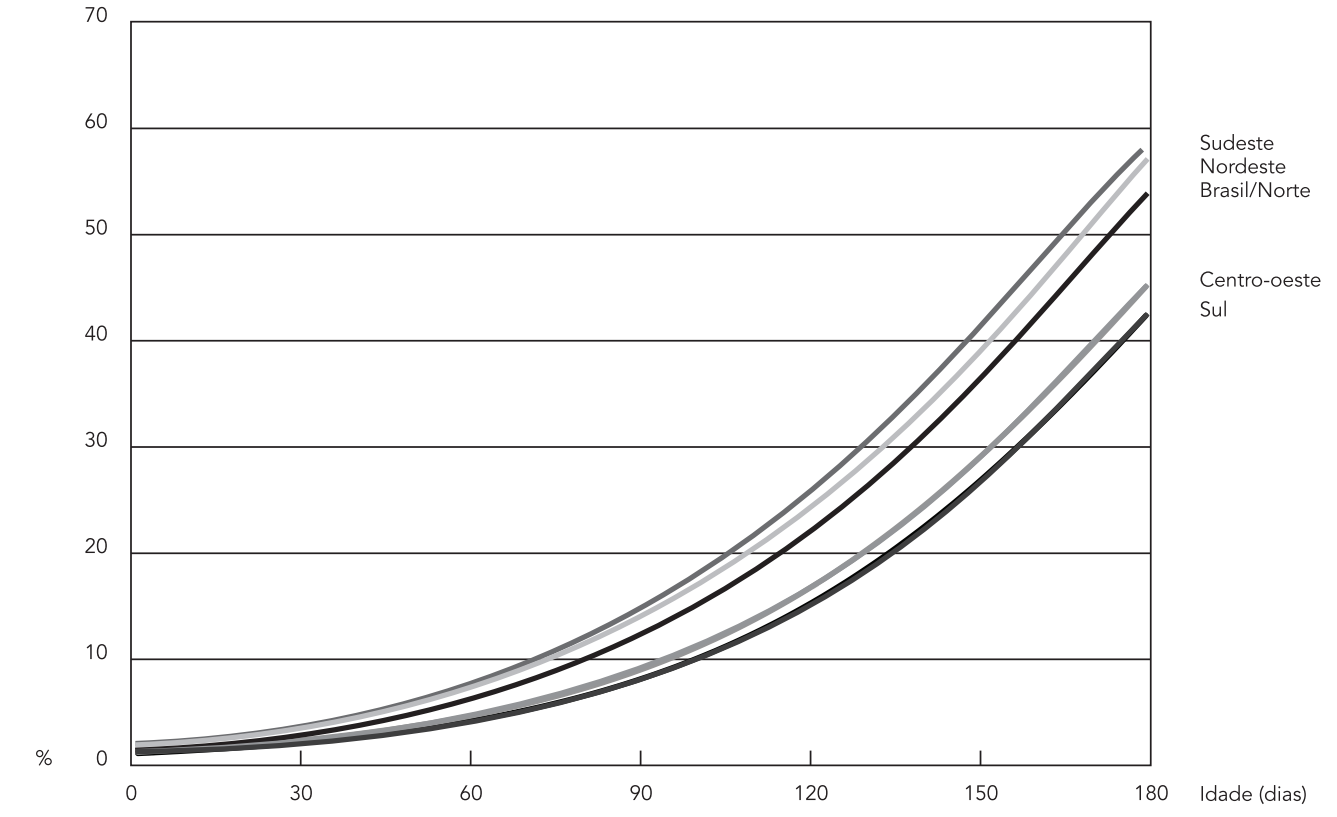


Estimativa da razão de prevalência bruta (RP bruta) e ajustada (RP ajustada) para a associação entre o consumo de chá, outros leites, sucos, mingau/papa e o conjunto das capitais distribuídas em regiões do país, em 2008.

\begin{tabular}{|c|c|c|c|c|c|c|c|c|}
\hline \multirow[t]{2}{*}{ Variáveis } & \multicolumn{2}{|c|}{ Chá } & \multicolumn{2}{|c|}{ Suco } & \multicolumn{2}{|c|}{ Outros leites } & \multicolumn{2}{|c|}{ Mingau/Papa } \\
\hline & $\begin{array}{c}\text { RP } \\
\text { bruta }\end{array}$ & $\begin{array}{c}\text { RP } \\
\text { ajustada }\end{array}$ & $\begin{array}{c}\text { RP } \\
\text { bruta }\end{array}$ & $\begin{array}{c}\text { RP } \\
\text { ajustada }\end{array}$ & $\begin{array}{c}\text { RP } \\
\text { bruta }\end{array}$ & $\begin{array}{c}\text { RP } \\
\text { ajustada }\end{array}$ & $\begin{array}{c}\text { RP } \\
\text { bruta }\end{array}$ & $\begin{array}{c}\text { RP } \\
\text { ajustada }\end{array}$ \\
\hline \multicolumn{9}{|l|}{ Região } \\
\hline Norte & 1,00 & 1,00 & 1,34 * & 1,42 * & 1,00 & 1,00 & 1,53 * & 1,67 * \\
\hline Nordeste & 1,65 * & 1,61 * & 1,34 * & 1,58 * & 1,34 * & 1,52 * & $2,61 *$ & 2,90 \\
\hline Centro-Oeste & 1,26 * & 1,24 * & 1,10 & 1,04 & 1,05 & 1,01 & 1,00 & 1,00 \\
\hline Sudeste & 1,84 * & 1,80 * & 1,65 * & 1,57 * & 1,58 * & 1,47 * & 1,61 * & 1,65 * \\
\hline Sul & 2,36 * & 2,34 * & 1,00 & 1,00 & 1,42 * & 1,31 * & 0,88 & 0,96 \\
\hline \multicolumn{9}{|l|}{ Sexo da criança } \\
\hline Masculino & 1,12 * & 1,18 * & 1,08 & 1,05 & 1,10 * & 1,15 * & 1,06 & 1,06 \\
\hline Feminino & 1,00 & 1,00 & 1,00 & 1,00 & 1,00 & 1,00 & 1,00 & 1,00 \\
\hline \multicolumn{9}{|l|}{ Baixo peso } \\
\hline $\operatorname{Sim}$ & 1,20 * & 1,20 & 0,90 & $\star \star$ & 1,28 * & 1,32 * & 1,14 & 1,15 \\
\hline Não & 1,00 & 1,00 & 1,00 & & 1,00 & 1,00 & 1,00 & 1,00 \\
\hline \multicolumn{9}{|l|}{ Primeiro filho } \\
\hline $\operatorname{Sim}$ & 1,20 * & 1,28 * & 1,23 * & 1,21 * & 1,09 * & 1,03 & 0,91 & 0,86 * \\
\hline Não & 1,00 & 1,00 & 1,00 & 1,00 & 1,00 & 1,00 & 1,00 & 1,00 \\
\hline \multicolumn{9}{|l|}{ Escolaridade da mãe } \\
\hline$<8$ & 1,33 * & 1,37 * & 1,02 & $\star \star$ & 0,99 & $\star \star$ & 1,55 * & 1,36 * \\
\hline$\geq 8$ & 1,00 & 1,00 & 1,00 & & 1,00 & & 1,00 & 1,00 \\
\hline \multicolumn{9}{|l|}{ Trabalho da mãe } \\
\hline Trabalha fora & 1,03 & $\star \star$ & 1,26 * & 1,37 * & $1,29 *$ & 1,30 * & 0,82 * & 1,04 \\
\hline Não trabalha fora & 1,00 & & 1,00 & 1,00 & 1,00 & 1,00 & 1,00 & 1,00 \\
\hline \multicolumn{9}{|l|}{ Faixa etária da mãe (anos) } \\
\hline$<20$ & 1,20 & 1,02 & 1,16 & 1,23 * & 0,92 & 1,05 & 1,54 * & $1,55 *$ \\
\hline $20-35$ & 1,01 & 1,00 & 0,99 & 1,01 & 0,89 * & 0,93 & 1,13 & 1,23 \\
\hline$>35$ & 1,00 & 1,00 & 1,00 & 1,00 & 1,00 & 1,00 & 1,00 & 1,00 \\
\hline \multicolumn{9}{|l|}{ Tipo de parto } \\
\hline Cesárea & 0,90 & 0,96 & 1,06 & $\star \star$ & 1,10 * & 1,07 * & 0,84 * & 0,90 \\
\hline Normal/Fórceps & 1,00 & $\star \star$ & 1,00 & $\star \star$ & 1,00 & 1,00 & 1,00 & 1,00 \\
\hline Idade da criança em dias & 1,01 & 0,99 & 1,02 * & 1,02 * & $1,01 *$ & 1,01 * & $1,01 *$ & $1,01 *$ \\
\hline
\end{tabular}

* $\mathrm{p}<0,05$

** Variável não entrou no modelo.

mar, no entanto, que houve uma boa representatividade da população de crianças menores de um ano nas capitais, haja vista a cobertura da segunda etapa da campanha de vacinação de 2008 ter sido superior a $80 \%$ em todas as capitais e média de 95\% para o Brasil (Sistema de Informação do Programa Nacional de Imunizações. http:// pni.datasus.gov.br, acessado em 10/Mar/2010). Além disso, no que se refere ao perfil da escolaridade materna (considerada neste estudo como proxy da situação socioeconômica), verifica-se semelhança entre a amostra analisada e os dados do Sistema de Informação de Nascidos Vi- vos em 2008, para mães com menos de 12 anos de estudo $(86,2 \%$ versus $83,8 \%$, respectivamente (Situação da base de dados nacional em 14/ Mar/2009. http://tabnet.datasus.gov.br/cgi/tab cgi.exe?sinascp/cnv/nvuf.def, acessado em 12/ Mar/2010). Assim, acreditamos que foi possível observar, por meio do conjunto das capitais brasileiras, a influência da região no consumo precoce de alimentos diferentes do leite materno. Esse achado pode ser útil na definição de estratégias diferenciadas para a promoção da alimentação complementar baseada nas características mais frequentes identificadas em cada região. 
As capitais da Região Sul, por exemplo, apresentam posição destacada no consumo de chá por crianças nos primeiros seis meses de vida, hábito bastante arraigado em alguns locais no Brasil, sobretudo no Sul. É possível que o clima frio da região favoreça a manutenção desse hábito, já que a bebida é tradicionalmente servida a uma temperatura morna; o chá é utilizado desde os primeiros dias de vida com finalidades terapêuticas, para alívio de cólicas e desconforto gerado por gases intestinais 20,21,22. Seu uso sofre, ainda, influência de alguns fatores, conforme estudo realizado no Sul do Brasil, no qual se verificou que a chance de a criança estar recebendo chá já no primeiro mês de vida era em torno de $50 \%$ maior se a mãe era adolescente e se coabitava com a avó materna 22 . Na presente pesquisa, ser adolescente não mostrou associação significativa com uso precoce de chás, embora, na análise bruta, as mães com menos de vinte anos tivessem probabilidade $20 \%$ maior de oferecer chá para os seus filhos nos primeiros seis meses de vida.

Se, por um lado, as capitais da Região Sul se destacaram pelo uso de chá, por outro, elas, juntamente com as capitais do Centro-oeste, destacaram-se pelo baixo consumo de suco em relação às capitais das outras regiões. $\mathrm{O}$ consumo precoce de suco foi mais comum em capitais das regiões mais quentes do país (Norte e Nordeste), onde existe abundância de variedades de frutos tropicais. Uma pesquisa revelou que, em $70 \%$ das crianças em Recife, Pernambuco, o suco foi o primeiro alimento a ser oferecido e, segundo os autores, poderia ser ainda o reflexo de antigas recomendações do Ministério da Saúde 23. O clima mais quente e a abundância de frutos regionais, no entanto, não explicam a alta prevalência do uso de suco em crianças menores de seis meses nas capitais da Região Sudeste. Outros fatores, possivelmente relacionados ao poder aquisitivo da população, devem estar contribuindo para que, por exemplo, um quarto das crianças do Estado de São Paulo já receba suco aos quatro meses 24. É interessante observar que as mães que trabalham fora tiveram uma chance maior de oferecer suco antes dos seis meses. Essas mães têm mais dificuldade em manter o AME e podem estar substituindo algumas mamadas ao seio por refeições com suco.

O consumo de outro leite nos primeiros seis meses de vida também foi mais comum nas capitais das regiões Nordeste e Sudeste, ratificando os achados de outro estudo recente 25. Este é um dado bastante preocupante, uma vez que o Nordeste é a região com as maiores taxas de mortalidade infantil do Brasil. São inúmeros os estudos que mostram a associação entre o uso precoce de outros leites e a morbimortalidade infantil 26,27,28.
Além disso, sabe-se que a introdução precoce de outro leite é um importante determinante do desmame precoce 22 , impedindo que as crianças recebam todos os demais benefícios do aleitamento materno, colocando a criança em risco maior de morbimortalidade.

Outro dado preocupante revelado pelo presente estudo é o fato de outros leites serem introduzidos precocemente com mais frequência em crianças com baixo peso de nascimento. Crianças nessa condição são, reconhecidamente, mais vulneráveis, sendo, portanto, as que mais se beneficiam com o leite materno 29,30.

As capitais da Região Nordeste se destacaram, ainda, pelo consumo precoce de mingaus/ papas, outro hábito considerado desnecessário, podendo ser prejudicial à saúde da criança. $\mathrm{O}$ consumo desses alimentos também foi bastante frequente nas capitais das regiões Norte e Sudeste. Em outros países, o hábito de introduzir precocemente alimentos sólidos/semissólidos é, igualmente, comum, sobretudo cereais, como no Canadá 31, nos Estados Unidos 30 e em diversos países europeus 12 .

Na revisão sistemática quanto à duração ideal do $\mathrm{AME}^{2}$, as crianças que receberam alimentação complementar a partir dos quatro meses não mostraram vantagens sob o ponto de vista nutricional; elas tiveram mais episódios de diarreia e internações por pneumonia, quando comparadas com as que se mantiveram em AME nos primeiros seis meses 2 . Em adição, já foi demonstrado que consumo precoce desses alimentos está associado a obesidade na infância e vida adulta e a doenças alérgicas 28,30.

As práticas alimentares são formadas predominantemente na infância, transmitidas por pais, familiares e cuidadores, sendo mantidas pela cultura, valores e crendices 1 . Em um país de dimensões continentais como o Brasil, é de se esperar que haja diferenças regionais nas práticas alimentares infantis, e este estudo confirmou que o consumo precoce de alimentos diferentes do leite materno por crianças menores de seis meses sofre forte influência da região em que estas residem. Esse achado deve ser levado em consideração na elaboração de estratégias que visem a reduzir essa prática, de modo que, por exemplo, o alto consumo de chá por crianças menores de seis meses nas capitais da Região Sul seja valorizado, assim como o consumo precoce de suco, outros leites e mingaus/papas nas capitais da Região Nordeste. É preciso, contudo, avançar no conhecimento sobre os fatores envolvidos no consumo precoce de alimentos diferentes do leite materno, para que possamos elaborar estratégias mais efetivas de melhoria do estado de saúde e nutrição da população infantil. 


\section{Resumo}

Objetivou-se avaliar a influência regional no consumo precoce de alimentos diferentes do leite materno em menores de seis meses residentes nas capitais brasileiras. Analisaram-se dados de 18.929 crianças da II Pesquisa de Prevalência de Aleitamento Materno nas Capitais Brasileiras - 2008. As frequências do consumo de chá, sucos, leite artificial e mingau/papa foram calculadas para as capitais das cinco regiões brasileiras. Curvas do consumo foram obtidas pela análise de logitos e estimativas das razões de prevalência (RP) por modelos de Poisson. O consumo de leite artificial foi maior quando comparado aos demais alimentos em todas as capitais. As capitais do Sul apresentaram a maior razão de prevalência para o consumo de chá $(R P=2,37)$ e as do Nordeste e Sudeste, para o consumo de outros tipos de leite $(R P=1,50$ e 1,47) e de suco $(R P=1,57$ e 1,55). Nas capitais do Nordeste, o consumo precoce de mingau/papa foi maior $(R P=3,0)$. A região tem influência no consumo precoce de alimentos, o que deve ser levado em consideração na elaboração de políticas públicas.

Suplementação Alimentar; Consumo de Alimentos; Lactente

\section{Colaboradores}

S. R. D. M. Saldiva participou do desenvolvimento da pesquisa, processamento de dados, elaboração do artigo, análise estatística dos dados, revisão da literatura e redação do texto. S. I. Venancio orientou as etapas de análise dos dados, redação e revisão do texto. A. G. C. Gouveia e A. L. S. Castro contribuíram na revisão da literatura, análise dos dados, construção das tabelas e redação do texto. M. M. L. Escuder participou do desenvolvimento da pesquisa e desenho amostral e da revisão do artigo. E. R. J. Giugliani participou do desenvolvimento da pesquisa, análise dos dados e revisão final do texto.

\section{Agradecimentos}

Agradecemos às coordenadoras estaduais e das capitais o esforço e dedicação empenhados na realização da pesquisa e digitação dos dados de seus municípios.

\section{Referências}

1. World Health Organization. Infant and Young Child Nutrition. Fity-Fourth World Health Assembly, WHA54.2. Geneva: World Health Organization; 2001.

2. Kramer MS, Kakuma R. The optimal duration of exclusive breastfeeding: a systematic review. Adv Exp Med Biol 2004; 554:63-77.

3. Giugliani ERJ, Victora CG. Alimentação complementar. J Pediatr (Rio J.) 2000; 76 Suppl 3:253-62.

4. Souza SL, Castro RM, Nogueira MI. Comportamento alimentar neonatal. Rev Bras Saúde Matern Infant 2003; 3:241-6.

5. Vieira GO, Silva LR, Vieira TO, Almeida JA, Cabral VA. Hábitos alimentares de crianças menores de 1 ano amamentadas e não amamentadas. J Pediatr (Rio J.) 2004; 80:411-6.

6. Departamento de Ações Programáticas e Estratégicas, Secretaria de Atenção à Saúde, Ministério da Saúde. II Pesquisa de Prevalência de Aleitamento Materno nas Capitais Brasileiras e Distrito Federal. Brasília, 2009. http://bvsms.saude.gov.br/bvs/pu blicacoes/pesquisa_prevalencia_aleitamento_ma terno.pdf (acessado em 18/Fev/2011).
7. Marchioni DML, Latorre MRDO, Szarfarc SC, Souza SB. Complementary feeding: study on prevalence of food intake in two Health Centers of São Paulo City. Arch Latinoam Nutr 2001; 51:161-6.

8. Oliveira LPM, Assis AMO, Gomes GSS, Prado MS, Barreto ML. Duração do aleitamento materno, regime alimentar e fatores associados segundo condições de vida em Salvador, Bahia, Brasil. Cad Saúde Pública 2005; 21:1519-30.

9. Brunken GS, Silva SM, França GVA, Escuder MML, Venancio SI. Fatores associados à interrupção precoce do aleitamento materno exclusivo e à introdução tardia da alimentação complementar no Centro-oeste brasileiro. J Pediatr (Rio J.) 2006; 82:445-51.

10. Alves CRL, Goulart EMA, Colosimo EA, Goulart LMHF. Fatores de risco para o desmame entre usuárias de uma unidade básica de saúde de Belo Horizonte, Minas Gerais, Brasil, entre 1980 e 2004. Cad Saúde Pública 2008; 24:1355-67. 
11. Bernardi JLD, Jordão RE, Barros Filho AA. Alimentação complementar de lactentes em uma cidade desenvolvida no contexto de um país em desenvolvimento. Rev Panam Salud Pública 2009; 26:405-11.

12. Schiess S, Grote V, Scaglioni S, Luque V, Martin F, Stolarczyk A, et al. Introduction of complementary feeding in 5 European countries. J Pediatr Gastroenterol Nutr 2010; 50:92-8.

13. Ford RP, Schluter PJ, Mitchel EA. Factors associated with the age of introduction of solids into the diet of New Zealand infants. New Zealand Cot Death Study Group. J Paediatr Child Health 1995; 31:469-72.

14. Lande B, Andersen LF, Baerug A, Trygg KU, LundLarsen $\mathrm{K}$, Veierod MB, et al. Infant feeding practices and associated factors in the first six months of life: the Norwegian infant nutrition survey. Acta Paediatr 2003; 92:152-61.

15. Venancio SI, Escuder MML, Saldiva SRDM, Giugliani ERJ. A prática de aleitamento materno nas capitais brasileiras e distrito federal: situação atual e avanços. J Pediatr (Rio J.) 2010; 86:317-24.

16. World Health Organization. Indicators for assessing infant and young child feeding practices: conclusions of a consensus meeting held 6-8 November 2007 in Washington D.C., USA. Geneva: World Health Organization; 2008.

17. Dewey KG, Brown KH. Update on technical issues concerning complementary feeding of young children in developing countries and implications for intervention programs. Food Nutr Bull 2003; 24:5-28.

18. Greer FR, Sicherer SH, Burks AW; Committee on Nutrition and Section on Allergy an Immunology. Effects of early nutritional interventions on the development of atopic disease in infants and children: the role of maternal dietary restriction, breastfeeding, timing of introduction of complementary foods, and hydrolyzed formulas. Pediatrics 2008; 121:183-91.

19. Agostini C, Decsi T, Fewtrell M, Goulet O, Kolacek S, Koletzko B, et al. Complementary feeding: a commentary by the ESPGHAN Committee on Nutrition. J Pediatr Gastroenterol Nutr 2008; 48:99-110.

20. Souza SL, Castro RM, Nogueira MI. Comportamento alimentar neonatal. Rev Bras Saúde Matern Infant 2003; 3:241-6.
21. Escobar AMU, Ogawa AR, Hiratsuka M, Kawashita MY, Teruya PY, Grisi S, et al. Tomikawa SO. Aleitamento materno e condições socioeconômicoculturais: fatores que levam ao desmame precoce. Rev Bras Saúde Matern. Infant 2002; 2:253-61.

22. Giugliani ER, Espírito Santo LC, Oliveira LD, Aerts D. Intake of water, herbal teas and non-breast milks during the first month of life: associated factors and impact on breastfeeding duration. Early Hum Dev 2008; 84:305-10.

23. Santos CS, Lima LS, Javorski C. Fatores que interferem na transição alimentar de crianças entre cinco e oito meses: investigação em serviço de puericultura do Recife, Brasil. Rev Bras Saúde Matern Infant 2007; 7:373-80.

24. Venancio SI, Saldiva SR, Mondini L, Levy RB, Escuder MM. Early interruption of exclusive breastfeeding and associated factors, state of São Paulo, Brazil. J Hum Lact 2008; 4:168-74.

25. Caetano MC, Ortiz TTO, Silva SGL, Souza FIS, Sarni ROS. Complementary feeding: inappropriate practices in infants. J Pediatr (Rio J.) 2010; 86:196-201.

26. Victora CG, Smith PH, Vaughan JP, Nobre LC, Lombardi C, Teixeira AM, et al. Evidence for protection by breastfeeding against infant deaths from infectious diseases in Brazil. Lancet 1987; 2:319-22.

27. César JA, Victora CG, Barros FC, Santos IS, Flores JA. Impact of breastfeeding on admission for pneumonia during post-neonatal period in Brazil: nested cases-control study. BMJ 1999; 318:1316-20.

28. McNiel ME, Labbok MH, Abrahams SW. What are the risks associated with formula feeding? A reanalysis and review. Birth 2010; 37:50-8.

29. Geib LTC, Fréu CM, Brandão M, Nunes ML. Determinantes sociais e biológicos da mortalidade infantil em coorte de base populacional em Passo Fundo, Rio Grande do Sul. Ciênc Saúde Coletiva 2010; 15:363-70.

30. Wu TC, Chen PH. Health consequences of nutrition in childhood and early infancy. Pediatr Neonatol 2009; 50:135-42.

31. Friel JK, Hanning RM, Isaak CA, Prowse D, Miller AC. Canadian infants' nutrients intakes from complementary foods during the first year of life. BMC Pediatr 2010; 17:10-43.

Recebido em 25/Abr/2011

Versão final reapresentada em 22/Ago/2011 Aprovado em 05/Set/2011 